\title{
Formation and Evolution of Black Holes in Galactic Nuclei and Star Clusters
}

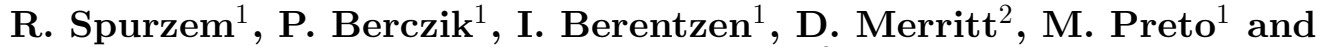 P. Amaro-Seoane ${ }^{3}$}

\author{
${ }^{1}$ Astronomisches Rechen-Institut, Zentrum für Astronomie Univ. Heidelberg, Mönchhofstr. \\ 12-14, 69120 Heidelberg, Germany \\ ${ }^{2}$ Dept. of Physics, 85 Lomb Memorial Drive, Rochester Institute of Technology, Rochester, NY \\ 14623-5604, USA \\ ${ }^{3}$ Max Planck Institute for Gravitational Physics (Albert Einstein Institute), Am Mühlenberg
} 1, 14476 Golm, Germany

\begin{abstract}
We study the formation, growth, and co-evolution of single and multiple supermassive black holes (SMBHs) and compact objects like neutron stars, white dwarfs, and stellar mass black holes in galactic nuclei and star clusters, focusing on the role of stellar dynamics. In this paper we focus on one exemplary topic out of a wider range of work done, the study of orbital parameters of binary black holes in galactic nuclei (binding energy, eccentricity, relativistic coalescence) as a function of initial parameters. In some cases the classical evolution of black hole binaries in dense stellar systems drives them to surprisingly high eccentricities, which is very exciting for the emission of gravitational waves and relativistic orbit shrinkage. Such results are interesting to the emerging field of gravitational wave astronomy, in relation to a number of ground and space based instruments designed to measure gravitational waves from astrophysical sources (VIRGO, Geo600, LIGO, LISA). Our models self-consistently cover the entire range from Newtonian dynamics to the relativistic coalescence of SMBH binaries.
\end{abstract}

Keywords. methods: numerical, n-body simulations, gravitational waves, black hole physics, galaxies: star clusters, nuclei

\section{Introduction}

SMBH formation and their interactions with their host galactic nuclei is an important ingredient for our understanding of galaxy formation and evolution in a cosmological context, e.g. for predictions of cosmic star formation histories or of SMBH demographics (to predict events which emit gravitational waves). If galaxies merge in the course of their evolution, there should be either many binary or even multiple black holes, or we have to find out what happens to black hole multiples in galactic nuclei, e.g. whether they come close enough together to merge under emission of gravitational waves, or whether they eject each other in gravitational slingshot. For numerical simulations of the problem all models depend on an unknown scaling behaviour, because the simulated particle number is not yet realistic due to limited computing power (Milosavljević \& Merritt 2001, Milosavljević \& Merritt 2003, Makino \& Funato 2004 and Berczik, Merritt \& Spurzem 2005). Dynamical modelling of non-spherical dense stellar systems (with and without central black holes) is even less developed than in the spherical case. Here we present a set of numerical models of the formation and evolution of binary black holes in rotating galactic nuclei. Since we are interested in the dynamical evolution of SMBH binaries in their final phases of evolution (the last parsec problem) we somehow abstract from the foregoing complex dynamics of galactic mergers. We assume that after some violent 
dynamic relaxation a typical initial situation consists of a spherical or axisymmetric coherent stellar system (galactic nucleus), where fluctuations in density and potential due to the galaxy merger have decayed, which is reasonable on an astrophysically short time scale of a few ten million years. The SMBHs, which were situated in the centre of each of the previously merged galaxies, are located at the boundary of the dense stellar core, some few hundred parsec apart. This situation is well observable (Komossa et al. 2003).

According to the standard theory, the subsequent evolution of the black holes is divided in three intergradient stages (Begelman, Blandford \& Rees 1980): 1. Dynamical friction causes an transfer of the black hole's kinetic energy to the surrounding field stars, the black holes spiral to the center where they form a binary. 2. While hardening, the effect of dynamical friction reduces and the evolution is dominated by superelastic scattering processes, that is the interaction with field stars closely encountering or intersecting the binary orbit, thereby increasing the binding energy. 3. Finally the black holes coalesce throughout the emission of gravitational radiation, potentially detectable by the planned space-based gravitational wave antennae LISA.

In this paper, the behavior of the orbital elements of a black hole binary in a dense stellar system is investigated in a self-consistent way from the beginning till the relativistic merger and its emission of gravitational waves. The evolution of the eccentricity has been discussed for some time e.g. Makino et al. (1993), Hemsendorf, Sigurdsson \& Spurzem (2002), Milosavljević \& Merritt (2001), Berczik, Merritt \& Spurzem (2005) and Makino \& Funato (2004). According to Peters \& Mathews (1963) and Peters (1964) the timescale of coalescence due to the emission of gravitational radiation is given by

$$
t_{g r}=\frac{5}{64} \frac{c^{5} a_{g r}^{4}}{G^{3} M_{1} M_{2}\left(M_{1}+M_{2}\right) F(e)}
$$

wherein $M_{1}, M_{2}$ denote the black hole masses, $a_{g r}$ the characteristic separation for gravitational wave emission, $G$ the gravitational constant, $c$ the speed of light and

$$
F(e)=\left(1-e^{2}\right)^{-7 / 2}\left(1+\frac{73}{24} e^{2}+\frac{37}{96} e^{4}\right)
$$

a function with strong dependence on the eccentricity $e$. Thus the coalescence time can shrink by several orders of magnitude if the eccentricity is high enough, resulting in a strengthened burst of gravitational radiation. Highly eccentric black hole binaries would represent appropriate candidates for forthcoming verification of gravitational radiation through the planned mission of the Laser Interferometer Space Antenna mission LISA.

\section{Numerical method, Initial Models}

The simulations have been performed using NBODY6++, a parallelized version of Aarseth's NBODY6 (Aarseth 1999, Spurzem 1999 and Aarseth 2003). The code includes a Hermite integration scheme, KS-regularization (Kustaanheimo \& Stiefel 1965) and the Ahmad-Cohen neighbour scheme (Ahmad \& Cohen 1973). No softening of the interaction potential of any two bodies is introduced; this allows an accurate treatment of the effects due to superelastic scattering events, which play a crucial part in black hole binary evolution and require a precise calculation of the trajectories throughout the interaction. The code and its parallel performance has been described in detail in this series and elsewhere (Spurzem 1999 and Khalisi et al. 2003). The survey has been carried out for a total particle number of up to $N=1000000$ including two massive black holes with $M_{1}=$ $M_{2}=0.01$ embedded in a dense stellar system of equal-mass particles $m_{*} \approx 1.0 \cdot 10^{-6}$. 
The total mass of the system is normalized to unity. The initial stellar distribution was taken from generalized King models with and without rotation (Einsel \& Spurzem 1999).

\section{Simulations}

\subsection{Newtonian Evolution of the binary black hole}

In the first evolutionary stage, each black hole individually suffers from dynamical friction with the surrounding low mass stars, which is the main process of losing energy. The role of dynamical friction decreases when a permanently bound state occurs, as the dynamical friction force acts preliminary on the motion of the now formed binary rather than on the individual black holes. Superelastic scattering events of field stars at the binary then become more and more important for the reduction of its energy. The process sustains an ongoing "hardening" of the binary (shrinking of semi-major axis and increase of energy) and also in many cases a high eccentricity. While the hardening rates are well understood (Sesana, Haardt \& Madau 2007 and Quinlan 1996) and do not depend much on the initial parameters of the preceding galactic merger, this is not as clear for the eccentricity.

In a spherically symmetric system the binary hardening would stall after a few crossing times, because loss-cone orbits of stars, which come close to the central SMBH binary will be depleted, and replenishment takes place only on a much longer relaxation time. This effect is more dramatic for systems with large particle number, because the relaxation time increases strongly with $N$ (Berczik, Merritt \& Spurzem 2005 and Berczik et al. 2006). It has been claimed that in real galaxies with their very large particle numbers therefore the SMBH binary will not reach relativistic coalescence. This situation was relaxed from two sides, first by a careful analysis of loss-cone refilling time scales combining direct $N$-body and Fokker-Planck models (Merritt, Mikkola \& Szell 2007), and by looking for a moderately rotating, axisymmetric galactic nucleus (Berczik et al. 2006), where the loss cone remains full even for large particle numbers. Since some degree of perturbation of a spherical model is quite natural for a remnant after galactic mergers, many of them might even be triaxial rather than axisymmetric, the stalling problem does not exist anymore.

\section{Relativistic Dynamics of Black Holes in Galactic Nuclei}

\subsection{Introduction}

Relativistic stellar dynamics is of paramount importance for the study of a number of subjects. For instance if we want to have a better understanding of what the constraints on alternatives to supermassive black holes are; in order to canvass the possibility of ruling out stellar clusters, one must do detailed analysis of the dynamics of relativistic clusters. Furthermore the dynamics of compact objects around SMBH and of multiple $\mathrm{SMBH}$ in galactic nuclei requires the inclusion of relativistic effects. Our current work deals with the evolution of two SMBHs, bound to each other, and looking at the phase when they get close enough to each other that relativistic corrections to Newtonian dynamics become important, which ultimately lead to gravitational radiation losses and coalescence.

Efforts to understand the dynamical evolution of a stellar cluster in which relativistic effects may be important have been already done by Lee (1987), Quinlan \& Shapiro (1989), Quinlan \& Shapiro (1990) and Lee (1993). In the earlier work $1 \mathcal{P N}$ and $2 \mathcal{P N}$ terms were neglected (Lee 1993) and the orbit-averaged formalism (Peters 1964) used. We describe here a method to deal with deviations from Newtonian dynamics more rigorously 


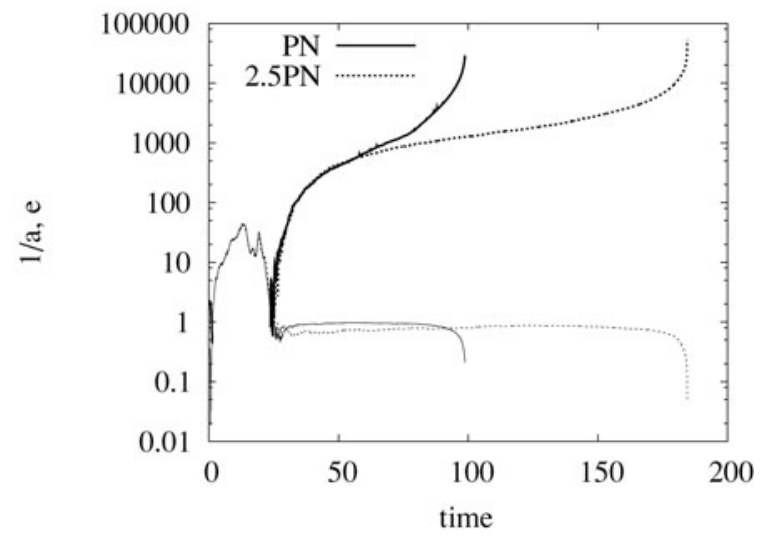

Figure 1. Effect of Post-Newtonian (PN) relativistic corrections on the dynamics of black hole binaries in galactic nuclei, plotted are inverse semi-major axis and eccentricity as a function of time. The solid line uses the full set of PN corrections, while the dotted line has been obtained by artificially only using the dissipative PN2.5 terms. Further details will be published elsewhere Berentzen et al. (2008, in preparation).

than in most existing literature (but compare Mikkola \& Merritt (2007), Aarseth (2007), which are on the same level of PN accuracy). We modified the NBODY6++ code to allow for post-Newtonian $(\mathcal{P N})$ effects of two particles getting very close to each other, implementing in it the $1 \mathcal{P N}, 2 \mathcal{P N}$ and $2.5 \mathcal{P N}$ corrections fully from Soffel (1989).

\subsection{Method: Direct summation NBODY with Post-Newtonian corrections}

The version of direct summation NBODY method we employed for the calculations, NBODy6++, includes the $K S$ regularisation. This means that when two particles are tightly bound to each other or the separation among them becomes smaller during a hyperbolic encounter, the couple becomes a candidate for regularisation in order to avoid problematical small individual time steps (Kustaanheimo \& Stiefel 1965). We modified this scheme to allow for relativistic corrections to the Newtonian forces by expanding the acceleration in a series of powers of $1 / c$ in the way given by Damour \& Dereulle (1981), Soffel (1989) and Kupi, Amaro-Seoane \& Spurzem (2006): We integrated our correcting terms as external forces into the two-body KS regularisation scheme.

\subsection{First results and Summary}

In Fig. 1 the impact of relativistic, Post-Newtonian dynamics to the separation of the binary black holes in our simulations is seen. The curve deviates from the Newtonian results when gravitational radiation losses set in and cause a sudden coalescence $(1 / a \rightarrow$ $\infty)$ at a finite time. Gravitational radiation losses are supported by the high eccentricity of the SMBH binary. It is interesting to note that the inclusion or exclusion of the conservative 1 and 2PN terms changes the coalescence time considerably. Details of these results will be published elsewhere Berentzen et al. (2008, in preparation).

Once the SMBH binary starts to dramatically lose binding energy due to gravitational radiation its orbital period will drop from a few thousand years to less than a year very quickly (timescale much shorter than the dynamical time scale in the galactic center, which defines our time units). Then the SMBH binary will enter the LISA band, i.e. its gravitational radiation will be detectable by LISA. LISA, Laser Interferometer Space Antenna, is a system of three space probes with laser interferometers to measure gravitational waves, see e.g. http://lisa.esa.int/. 
We have shown that supermassive black hole binaries in galactic nuclei can overcome the stalling barrier and will reach the relativistic coalescence phase in a timescale shorter than the age of the universe. A gravitational wave signal for the LISA satellite from these SMBH binaries is expected, in particular due to the high eccentricity of the SMBH binary when entering the relativistic coalescence phase. Our models cover self-consistently the transition from the Newtonian dynamics to the situation when relativistic, PostNewtonian $(\mathrm{PN})$ corrections start to influence the relative SMBH motion (Preto et al. (2008, in preparation)).

\section{Acknowledgements}

Computing time at NIC Jülich on the IBM Jump is acknowledged. Financial support comes partly from Volkswagenstiftung, German Science Foundation (DFG) via Schwerpunktprogramm 1177 (Project ID Sp 345/17-1) "Black Holes Witnesses of Cosmic History". It is a pleasure to acknowledge many enlightening discussions and support by Sverre Aarseth, very useful interactions about relativistic dynamics with A. Gopakumar and G. Schäfer.

\section{References}

Aarseth S. J. 1999, PASP 111, 1333

Aarseth, S. J. 2003, in: Gravitational N-body simulations, Cambridge University Press, Cambridge, p. 173

Aarseth, S. J. 2007, MNRAS 378, 285

Ahmad, A. \& Cohen, L. 1973, Journal of Computational Physics 12, 349

Begelman, M. C., Blandford, R. D., \& Rees, M. J. 1980, Nature 287, 307

Berczik, P., Merritt, D., \& Spurzem, R. 2005, ApJ 633, 680

Berczik, P., Merritt, D., Spurzem, R., \& Bischof, H.-P. 2006, ApJ (Letters) 642, L21

Damour, T. \& Dereulle, N. 1987, Phys. Rev. 87, 81

Einsel, C. \& Spurzem, R. 1999, MNRAS 302, 81

Hemsendorf, M., Sigurdsson, S., \& Spurzem R. 2002, ApJ 581, 1256

Khalisi, E., Omarov, C. T., Spurzem, R., Giersz, M., \& Lin, D. N. C. 2003, in: High Performance Computing in Science and Engineering, Springer Vlg., p. 71

Komossa, S., Burwitz, V., Hasinger, G., Predehl, P., Kaastra, J. S. \& Ikebe, Y. 2003, ApJ (Letters) 582, L15

Kupi, G., Amaro-Seoane, P., \& Spurzem, R. 2006, MNRAS 371, 45

Kustaanheimo, P. \& Stiefel E. 1965, Journal für die reine und angewandte Mathematik 218, 204

Lee, H. M. 1987, ApJ 319, 801

Lee, M. H. 1993 AJ 418, 147

Makino, J., Fukushige, T., Okumura, S. K., \& Ebisuzaki T. 1993, PASJ 45, 303

Makino, J. \& Funato, Y. 2004, ApJ 602, 93

Mikkola, S. \& Merritt, D. 2007, arXiv:0709.3367

Merritt, D., Mikkola, S., \& Szell, A. 2007, arXiv:0705.2745

Milosavljević M. \& Merritt D. 2001, ApJ 563, 34

Milosavljević, M. \& Merritt, D. 2003, ApJ 596, 860

Peters, P. C. 1964, Phys. Rev. 136, 1224

Peters, P. C. \& Mathews, J. 1963 Phys. Rev. 131, 435

Quinlan, G. D. 1996, New Astron. 1, 35

Quinlan, G. D. \& Shapiro, S. L. 1989 AJ 343, 725

Quinlan, G. D. \& Shapiro, S. L. 1990, AJ 356, 483

Sesana, A., Haardt, F., \& Madau, P. 2007, AJ 660, 546

Soffel, M. H. 1989, in: Relativity in Astrometry, Celestial Mechanics and Geodesy, SpringerVerlag Berlin, Heidelberg, New York

Spurzem, R. 1999, Journal of Computational and Applied Mathematics 109, 407 